\title{
Asymmetric Dimethylarginine in the Maternal and Fetal Circulation in Preeclampsia
}

\author{
KRISTIN BRAEKKE, PER M. UELAND, NINA K. HARSEM, AND ANNE CATHRINE STAFF
}

Departments of Paediatrics [K.B.] and Obstetrics and Gynaecology [A.C.S., N.K.H.], Ulleval University Hospital, Oslo 0407, Norway; Department of Internal Medicine [P.M.U.], University of Bergen, Bergen 5021, Norway; Faculty of Medicine [A.C.S.], University of Oslo, Oslo 0407, Norway

\begin{abstract}
Preeclampsia is a leading cause of intrauterine growth restriction and preterm birth. Endothelial dysfunction is the common final pathway leading to clinical signs of preeclampsia including hypertension and proteinuria. Asymmetric dimethylarginine (ADMA) is an endogenous inhibitor of NOS and induces endothelial dysfunction by reversibly inhibiting NO production from L-arginine. The purpose of this study was to investigate maternal and fetal concentrations of ADMA, L-arginine, and symmetric dimethylarginine (SDMA). Women with preeclampsia $(n=47)$ and controls $(n=$ 51) who gave birth by cesarean section were included in the study. We analyzed the maternal plasma and umbilical vein and artery plasma. We found that not only maternal concentrations of ADMA and SDMA but also L-arginine were significantly higher in women with preeclampsia than in controls. In fetal samples, only SDMA concentrations were higher in the preeclampsia group than in controls. The median ADMA concentration was three times higher in the fetal circulation than in the maternal circulation, but there was no difference between the preeclampsia group and the control group, and the veno-arterious gradient indicated that the placenta was the source of ADMA. (Pediatr Res 66: 411-415, 2009)
\end{abstract}

$\mathrm{P}$ reeclampsia is a common pregnancy complication associated with increased risk of preterm birth and fetal growth restriction (1). The exact etiology of preeclampsia is not known, and hence no definitive preventative measures or treatments are available.

Endothelial dysfunction is the common final pathway leading to the clinical signs of preeclampsia including hypertension and proteinuria. It is not known to what extent endothelial dysfunction also affects the infant. Increased concentrations of biomarkers for preeclampsia in the maternal circulation are usually not reflected in the infant. Previously, our group has shown that newborns from preeclamptic pregnancies did not differ from those born after uncomplicated pregnancies with respect to circulating biomarkers of inflammation (CRP and calprotectin) (2), and oxidative stress (3), but newborns from preeclamptic pregnancies had elevated concentrations of homocysteine (4).

In women with previous preeclampsia, there is an increased risk for cardiovascular disease later in life (5) and an increased risk of hypertension has also been found for their offspring $(6,7)$.

Received January 8, 2009; accepted May 26, 2009.

Correspondence: Kristin Braekke, M.D., Ph.D., Department of Paediatrics, Ulleval University Hospital, Kirkeveien 166, 0407 Oslo, Norway; e-mail: kbre@uus.no; krb@broadpark.no
It is not clear whether the increased risk in the offspring is caused by the intrauterine environment, shared genes, or impaired intrauterine growth. In epidemiologic and animal studies, low birth weight by itself is associated with an increased risk of cardiovascular disease and metabolic syndrome (8).

$\mathrm{NO}$ is essential for vascular tone and endothelial function, and elevated circulating concentrations of asymmetric dimethylarginine (ADMA) are associated with reduced vasodilation and endothelial dysfunction (9). ADMA is an endogenous inhibitor of NOS and induces endothelial dysfunction by reversibly inhibiting NO production from $\mathrm{L}$-arginine. The ratio of L-arginine to ADMA is a key determinant of NOS activity and is a useful index for evaluating the effects of ADMA $(10,11)$. Symmetric dimethylarginine (SDMA), an ADMA isomer, has no effect on NOS, but it is a competitor of $\mathrm{L}$-arginine transport, and may therefore interfere with $\mathrm{NO}$ production and endothelial function (12). Inhibition of NO by ADMA has been proposed to be a unifying mechanism for endothelial dysfunction caused by elevated concentrations of homocysteine (13).

The fact that pregnant women have lower circulating ADMA concentrations than nonpregnant women suggests that ADMA may play a role in vascular dilatation and blood pressure regulation during pregnancy (14). There have been discrepant findings of elevated (15) and unchanged $(16,17)$ ADMA concentrations in the maternal circulation in women with preeclampsia compared with women with uncomplicated pregnancies. Elevated maternal ADMA concentrations have been found preceding the clinical symptoms of preeclampsia $(18,19)$ suggesting that ADMA could have a role in the pathogenesis of preeclampsia.

There are sparse data on fetal concentrations of ADMA and its associated molecules in pregnancy and preeclampsia (20). The aim of this study was to simultaneously explore maternal and fetal concentrations of L-arginine, ADMA, and SDMA in preeclampsia and possible associations of these measurements with gestational characteristics.

\section{METHODS}

Protocol. In this study, we examined women with preeclampsia $(n=47)$ and women with uncomplicated pregnancies (controls, $n=51$ ). All were

Abbreviations: ADMA, asymmetric dimethylarginine; SDMA, symmetric dimethylarginine 
singleton pregnancies delivered by cesarean section, providing maternal and fetal (umbilical cord) blood. At the time of blood sampling, all women were fasting, they were not in active labor, and none had rupture of membranes or signs of infection. Cesarean section was performed in the preeclamptic women because vaginal delivery was not considered appropriate because of disease progression and/or unfavourable cervical ripening. Controls were healthy, normotensive women undergoing cesarean section because of breech presentation, previous cesarean section, or for psychological reasons. This study was part of an ongoing biobank collection at Ulleval University Hospital. Findings for homocysteine and creatinine levels in this cohort have been reported in a previous publication (4).

Preeclampsia was defined as a rise in blood pressure above 140/90 after gestational week 20 on at least two occasions $6 \mathrm{~h}$ apart in a previously normotensive woman, combined with proteinuria. Proteinuria was defined as a protein dipstick reading equal to or higher than $1+$ on more than one midstream urine sample $6 \mathrm{~h}$ apart or a 24-h urine excretion of $\geq 0.3 \mathrm{~g}$ protein in the absence of urinary infection. Severe preeclampsia included patients with systolic blood pressure $\geq 160 \mathrm{~mm} \mathrm{Hg}$ (21).

Pregnancy duration was calculated on the basis of routine ultrasonographic screening between gestational weeks 17 and 20. Medical history and obstetrical information were obtained from medical records and from the patients' national pregnancy charts, as well as by interviewing the patients. The infants' weight percentiles were estimated from weight curves based on longitudinal ultrasonographic measurements of a population of healthy pregnant Norwegian women (22). Infants below the 10th percentile were defined as small for gestational age (SGA). The study protocol was approved by the Regional Committee of Medical Research Ethics in Eastern Norway, and informed written consent was obtained from each woman.

Biologic samples. Maternal blood samples were obtained before cesarean section. The umbilical cord was double clamped near the placenta and near the infant; then, immediately after delivery of the placenta, blood from the umbilical arteries and blood from the umbilical vein were sampled separately into EDTA-containing vials. Samples were kept on ice for a maximum of 30 min and centrifuged at $4^{\circ} \mathrm{C}$ for $10 \mathrm{~min}$ at $2000 \times \mathrm{g}$, and plasma was stored in aliquots at $-76^{\circ} \mathrm{C}$ until analysis. Laboratory personnel were blinded to clinical information.

Biochemical analyses. L-Arginine, ADMA, and SDMA were measured in EDTA plasma using normal-phase liquid chromatography and tandem mass spectrometry (LC-MS/MS) (23). The within- and between-day imprecision $\mathrm{CV}$ s for all three metabolites were between 4 and $6 \%$. Serum uric acid was measured as part of the routine screen only in preeclamptic patients; the results were obtained from the women's clinical records.

Statistics. As not all the values were normally distributed, medians are reported and the Mann-Whitney $U$ test was used for testing differences between groups, and the Wilcoxon Signed Rank Test was used for related samples (comparing concentrations in the umbilical vein and artery). Statistical analyses were performed using the Statistical Package for the Social Sciences (version 14.0; SPSS Inc., Chicago, IL). A probability level of $<0.05$ was considered statistically significant.

\section{RESULTS}

Clinical characteristics are presented in Table 1. Median gestational age, birth weight, and infant birth weight percen-

Table 1. Characteristics of the study population

\begin{tabular}{lccc}
\hline & $\begin{array}{c}\text { Preeclampsia } \\
(n=47)\end{array}$ & $\begin{array}{c}\text { Controls } \\
(n=51)\end{array}$ & $p$ \\
\hline Maternal age, y & $31.5(19-42)$ & $32.5(21-44)$ & 0.76 \\
BMI before & $24.1(18.9-41.1)$ & $22.5(17.4-29.4)$ & $0.01^{*}$ \\
$\quad$ pregnancy, $\mathrm{kg} / \mathrm{m}^{2}$ & & & \\
Gestational age, wk & $32.9(24.9-38.7)$ & $38.7(34.4-41.7)$ & $<0.001^{*}$ \\
BP at delivery, & $160(145-220)$ & $120(90-140)$ & $<0.001^{*}$ \\
$\quad$ systolic & $102(90-119)$ & $70(60-92)$ & $<0.001^{*}$ \\
$\begin{array}{l}\text { BP at delivery, } \\
\text { diastolic }\end{array}$ & $1.72(0.87-3.86)$ & $3.47(2.80-4.68)$ & $<0.001^{*}$ \\
Birth weight, kg & $7(1-92)$ & $69(6-99)$ & $<0.001^{*}$ \\
Birth weight percentile & 60 & 6 & $<0.001^{*}$ \\
Percent SGA & & 6
\end{tabular}

Values are presented as medians and range (minimum and maximum values), except for percent SGA.

$* p<0.05$. tile were lower for the preeclampsia group compared with the uncomplicated pregnancy group, whereas maternal BMI at start of pregnancy was higher in the preeclampsia group.

Because of the technical problems in sampling not all analyses were done in both the fetal and maternal blood samples; the exact numbers are given in Table 2. Samples from all three compartments were obtained for 47 cases (22 preeclampsia cases and 25 controls). In this sample set, the results were essentially the same as for the total number of samples.

Concentrations of L-arginine, ADMA, SDMA, and relevant ratios for all three compartments (maternal and fetal, both umbilical vein and umbilical artery) are shown in Table 2.

Metabolites in maternal circulation. Median maternal concentrations of L-arginine, ADMA, and SDMA were higher $(16,13$, and $30 \%$, respectively), whereas the maternal ADMA/ SDMA ratio was $12 \%$ lower in the preeclampsia group compared with the uncomplicated pregnancy group. There was no statistically significant difference in the maternal L-arginine/ ADMA ratio between preeclampsia patients and controls.

Maternal L-arginine, ADMA, and SDMA concentrations were highly correlated with each other in the preeclampsia group, but not in controls (Table 3).

Metabolites in fetal circulation. There was no difference in L-arginine or ADMA between infants in the preeclampsia and control groups in either the umbilical vein or the umbilical artery samples (Table 2).

Concentrations of L-arginine and ADMA were significantly higher in the umbilical vein than in the umbilical artery (both $p<0.001$ ), for both the preeclampsia and control groups, and there was a strong positive correlation between umbilical vein and artery concentrations for both L-arginine $(r=0.88, p<$ $0.001)$ and ADMA $(r=0.63, p<0.001)$. Also, a higher median SDMA concentration was found in the umbilical vein than in the umbilical artery $(p=0.001)$ in the newborns that provided paired artery and vein samples (controls $n=31$, preeclampsia $n=22$ ). Also, SDMA levels in the umbilical vein and artery were highly correlated $(r=0.87, p<0.001)$.

Relation between metabolites in the maternal and fetal circulation. The median ADMA concentration was almost three times higher in umbilical vein plasma than in maternal plasma. As with most amino acids, the L-arginine concentration was higher in the fetal circulation than in the maternal circulation, but the significantly higher maternal L-arginine concentration found in preeclampsia patients relative to controls was not reflected on the fetal side (in neither the umbilical vein nor the umbilical artery samples). In controls, the median concentration of L-arginine was twofold higher in the umbilical vein than in the maternal plasma. This ratio was significantly lower for the preeclampsia group $(p<0.001)$. The $\mathrm{L}$-arginine/ADMA ratio in the umbilical vein was $15 \%$ lower in the preeclampsia group compared with the control group.

Fetal and maternal concentrations were positively correlated for ADMA, L-arginine, and SDMA ( $\mathrm{r}=0.5-0.7, p<$ 0.001 for all). This applied to both the preeclampsia and control groups, except for ADMA in the control group for which there was no correlation between the maternal and umbilical artery concentrations $(r=0.09, p=0.68)$. Because 
Table 2. Median values and interquartile ranges for the measured variables and relevant ratios. Creatinine is included

\begin{tabular}{|c|c|c|c|c|c|c|c|c|c|}
\hline \multirow[b]{2}{*}{ Variable } & \multicolumn{3}{|c|}{ Maternal } & \multicolumn{3}{|c|}{ Umbilical vein } & \multicolumn{3}{|c|}{ Umbilical artery } \\
\hline & $\begin{array}{l}\text { Preeclampsia } \\
\quad(n=43)\end{array}$ & $\begin{array}{l}\text { Controls } \\
(n=40)\end{array}$ & $p$ & $\begin{array}{l}\text { Preeclampsia } \\
\quad(\mathrm{n}=38)\end{array}$ & $\begin{array}{l}\text { Controls } \\
(n=45)\end{array}$ & $p$ & $\begin{array}{l}\text { Preeclampsia } \\
\quad(n=25)\end{array}$ & $\begin{array}{l}\text { Controls } \\
(n=31)\end{array}$ & $p$ \\
\hline $\begin{array}{l}\text { L-arginine } \\
\qquad(\mu \mathrm{mol} / \mathrm{L})\end{array}$ & $45.4(37.7-54.4)$ & $39.1(34.6-45.2)$ & $0.006^{*}$ & $77.3(61.3-86.9)$ & $85.3(69.7-95.0)$ & 0.13 & $65.7(51.8-84.0)$ & $69.3(63.0-88.7)$ & 0.08 \\
\hline $\begin{array}{l}\text { ADMA } \\
\qquad(\mu \mathrm{mol} / \mathrm{L})\end{array}$ & $0.44(0.38-0.50)$ & $0.39(0.33-0.43)$ & $0.003 *$ & $1.27(1.15-1.38)$ & $1.28(1.18-1.38)$ & 0.67 & $1.18(1.07-1.36)$ & $1.21(1.10-1.31)$ & 0.93 \\
\hline $\begin{array}{l}\text { SDMA } \\
\qquad(\mu \mathrm{mol} / \mathrm{L})\end{array}$ & $0.69(0.59-0.75)$ & $0.53(0.46-0.59)$ & $<0.001 *$ & $1.98(1.80-2.40)$ & $1.69(1.48-2.01)$ & $<0.001^{*}$ & $2.14(1.78-2.32)$ & $1.61(1.39-1.84)$ & $0.001^{*}$ \\
\hline $\begin{array}{c}\text { L-arginine/ } \\
\text { ADMA }\end{array}$ & $105.8(91-126)$ & $106.5(90-121)$ & 0.78 & $57.4(50.8-68.3)$ & $66.0(56.3-72.9)$ & $0.03 *$ & $55.1(48.1-64.0)$ & $62.1(54.3-72.6)$ & 0.07 \\
\hline $\begin{array}{l}\text { ADMA/ } \\
\text { SDMA }\end{array}$ & $0.64(0.54-0.77)$ & $0.73(0.59-0.87)$ & $0.026^{*}$ & $0.63(0.51-0.75)$ & $0.74(0.65-0.86)$ & $0.002 *$ & $0.62(0.49-0.71)$ & $0.77(0.64-0.86)$ & $<0.001 *$ \\
\hline $\begin{array}{l}\text { Creatinine } \\
\qquad(\mu \mathrm{mol} / \mathrm{L})\end{array}$ & $59.9(52.2-69.8)$ & $51.4(45.9-58.6)$ & $<0.001 *$ & $59.7(53.0-66.7)$ & $51.1(47.6-57.4)$ & $0.001 *$ & $60.6(52.1-69.7)$ & $51.0(47.3-56.9)$ & $0.005^{*}$ \\
\hline
\end{tabular}

$* p<0.05$

Table 3. Correlations between maternal plasma concentrations of $L$-arginine, ADMA, SDMA, homocysteine, and creatinine

\begin{tabular}{lllll}
\hline & L-arginine & ADMA & SDMA & Homocysteine \\
\hline Controls & & & & \\
ADMA & 0.21 & & & \\
SDMA & 0.003 & 0.21 & & \\
Homocysteine & 0.10 & 0.18 & 0.24 & \\
Creatinine & 0.22 & 0.18 & $0.40^{*}$ & $0.41^{*}$ \\
Preeclampsia & & & & \\
ADMA & $0.66^{*}$ & & & \\
SDMA & $0.35^{*}$ & $0.38^{*}$ & & \\
Homocysteine & 0.09 & 0.25 & $0.31^{*}$ & \\
Creatinine & 0.26 & 0.19 & $0.59^{*}$ & 0.15 \\
\hline
\end{tabular}

$* p<0.05$.

of the high correlation between maternal and fetal concentrations, only correlations for maternal values are presented in Table 3.

Metabolites and clinical outcomes. No correlation was found between maternal ADMA (or SDMA and L-arginine) and maternal clinical characteristics such as prepregnancy BMI, maternal age at delivery, or maximum systolic or diastolic blood pressure, either in the preeclampsia group or in the control group (data not shown).

We did not find any significant difference in the concentrations of maternal or fetal L-arginine, ADMA, or SDMA between patients with severe $(n=34)$ and nonsevere $(n=10)$ preeclampsia. Also, there was not a statistically significant difference in the median concentrations of maternal or fetal L-arginine, ADMA, or SDMA between the preeclampsia groups delivered before or after week 34 (data not shown).

We found an inverse correlation between the maternal L-arginine/ADMA ratio and birth weight percentile $(r=$ $-0.39, p=0.01$ ), whereas no association was found between infant weight percentile and ADMA or any of the other metabolites presented in Table 2.

Maternal SDMA was correlated with creatinine both in the preeclampsia and control groups, whereas no correlation was found between maternal ADMA and creatinine (Table 3). The median maternal uric acid concentration was above normal for the preeclampsia group (median $400 \mu \mathrm{M}$, interquartile range $337-445 \mu \mathrm{M})$. Uric acid is often elevated in preeclamptic women, and in our study, it was positively correlated with
$\operatorname{ADMA}(r=0.32, p=0.04)$ and $\operatorname{SDMA}(r=0.39, p=0.01)$, but not with L-arginine $(r=0.19, p=0.24)$ in the preeclampsia group (uric acid was not measured in the control group).

\section{DISCUSSION}

In this study, we found that ADMA concentrations were slightly elevated in maternal, but not in fetal (umbilical vein and artery), plasma in preeclampsia patients compared with controls, whereas SDMA was significantly elevated in all three compartments in preeclampsia. We found a positive correlation between maternal concentrations of creatinine and SDMA, but not between creatinine and ADMA. This is consistent with SDMA being eliminated strictly by renal excretion, whereas ADMA is mainly metabolized by the enzyme dimethylarginine dimethylaminohydrolase (DDAH) (24), and it has been hypothesized that ADMA concentration is dependent on the activity of DDAH (25). Although maternal creatinine concentrations for the preeclampsia group were well within the normal range, median plasma creatinine was elevated in the preeclampsia group compared with the control group (Table 2), which is indicative of generalized endothelial dysfunction in preeclampsia, with a typical histologic finding of "glomerular endotheliosis" in the kidneys (26). The elevated concentrations of SDMA throughout all compartments in preeclampsia could be regarded as a marker of mild renal dysfunction in preeclampsia. We did not address the question of whether this elevated SDMA concentration influences NO through competition for the arginine y-transporter system in this study.

Circulating SDMA concentrations in preeclampsia have previously been explored in a few studies $(17,27)$. Ellis et al. reported elevated maternal SDMA in preeclampsia but, unlike our findings, they found that SDMA was correlated with arterial blood pressure and disease severity (27). In a study of Colombian women, Maas et al. (17) did not find that maternal ADMA or SDMA was elevated in preeclampsia. In contrast to our study, the majority of their participants had moderate preeclampsia, whereas ADMA levels seemed to be higher in their severe preeclampsia group (19 of 67), but it was not stated whether this was a significant difference (17).

Elevated maternal L-arginine concentrations have been previously found in preeclampsia (18), as we found in our study, 
but other studies have shown unchanged $(15,17,19)$ or lower concentrations (16) compared with controls. It has been argued that the L-arginine/ADMA ratio, rather than ADMA levels, is the key determinant of NOS activity (10), which is regarded as a useful index for interpretation of ADMA's effects (11). The L-arginine/ADMA ratio may be more important than ADMA levels, but in our study, there was no difference in the maternal plasma L-arginine/ADMA ratio between preeclampsia patients and controls. Therefore, an inhibitory effect of elevated ADMA on NOS in preeclampsia may be blunted because of concomitant elevation of the NO precursor L-arginine.

We found an unexpected negative correlation between the maternal L-arginine/ADMA ratio and birth weight percentile, suggesting that the offspring of preeclamptic pregnancies with more severe disease including placental insufficiency and low birth weight percentile, have the most "beneficial" L-arginine/ ADMA ratio. Elevated concentrations of maternal plasma amino acids have previously been reported in preeclampsia (28), and the higher L-arginine/ADMA ratio in preeclampsia could be a proxy for disease severity.

Both animal and human studies have suggested that ADMA could be a link between elevated homocysteine concentrations and endothelial dysfunction as a cardiovascular risk factor (13). We have previously shown in the same biobank cohort that homocysteine is elevated in preeclampsia both in the maternal and fetal circulation (4). However, we found no correlation between ADMA and homocysteine in our preeclampsia or control group (Table 3), which is in accordance with a study by Herrmann et al. (29).

The role of ADMA in normal pregnancies has been explored in a few studies. Maeda et al. (30) found maternal concentrations of ADMA to be lower in normal pregnancies than in nonpregnant women and suggested that ADMA is important for vasodilation in pregnancy. Recently, Saarelainen et al. (31) have expressed doubt regarding the role of ADMA as a key regulator of blood pressure in normal pregnancy, because their study demonstrated only subtle alterations of ADMA and no correlation between endotheliumdependent vasodilation markers and maternal serum ADMA or L-arginine concentrations.

The role of NO inhibition in preeclampsia is not entirely clear, and the evidence that endothelial dysfunction is induced by ADMA inhibition or low L-arginine is not unequivocal. Despite this, L-arginine supplementation to prevent preeclampsia has been tried in several studies. Oral L-arginine supplementation was investigated by our group; the intervention did not alter blood pressure in preeclampsia (32). A recent review found that evidence is still insufficient to draw reliable conclusions as to whether L-arginine can prevent preeclampsia or its complications (33). Interestingly, a study involving dietary supplementation with $3 \mathrm{~g} / \mathrm{d}$ L-arginine found no difference in biochemical markers, but supplementation significantly decreased maternal blood pressure and improved fetal outcome $(34,35)$. Conceivably, in studies evaluating Larginine and other nutritional supplements (i.e., calcium, antioxidants), the outcomes depend on the general nutritional status of the group. In some populations where insufficient nutrition is more prevalent, it is more likely that the incidence of preeclampsia could be reduced by supplementing the deficient nutrients (36).

In our study, fetal ADMA concentrations (in the umbilical vein and artery) did not differ between preeclampsia patients and controls, and this is consistent with a recently published study (20). This is also in accordance with our previous findings that in preeclampsia, elevated maternal levels of markers of inflammation and oxidative stress are generally not reflected in the fetal circulation $(2,3)$.

For ADMA and L-arginine concentrations, there were consistent veno-arterial differences, with higher levels in the umbilical vein compared with the umbilical artery, suggesting that these metabolites are mainly placentally derived, not fetally, which is consistent with a study performed in normal infants (37). ADMA and SDMA may be derived from the placenta, but may also be concentrated from the maternal plasma; it is not possible to discern between these possibilities from this study. The very high concentration of ADMA in the fetal circulation is an enigma, and it has been hypothesized that it is a compensatory mechanism to stabilize very high NO concentrations in the fetoplacental unit $(30,37)$.

A strength of this study is that the included subjects are clinically well defined. As our preeclampsia group was delivered by cesarean section, they clinically had more severe preeclampsia than if vaginal deliveries were also included. Our preeclampsia group is therefore not representative of all women with preeclampsia, but is possibly the most interesting and important group to study, as severe preeclampsia and premature delivery have serious consequences for the infant. Our model, with concomitant sampling from all three compartments (maternal, placental, and fetal), allows us to speculate on the origin of metabolites and their correlations.

It is possible that some of the discrepant findings in studies on ADMA are because of the differences in laboratory methods. In this study, a well-described and validated LC-MS/MS method was used (23), which has the advantage of differentiating between ADMA and SDMA with high specificity (24).

One limitation of our study is that the median gestational age was different in preeclampsia patients and controls. It is difficult to match for gestational age when sampling fetal blood between the often prematurely delivered preeclamptic patients and women with uncomplicated deliveries, because cesarean section is not performed preterm on healthy women. However, in a longitudinal study of ADMA in normal pregnancies (14), maternal concentrations of ADMA increased slightly with increasing gestational age, which in our study means that the difference in ADMA concentration between preeclampsia patients and controls could be underestimated.

In summary, ADMA is increased in the maternal circulation in preeclampsia, but so is L-arginine, possibly attenuating the effects on NOS. Elevated SDMA levels are possibly because of slightly reduced renal clearance. ADMA is three times higher in the fetal circulation than in the maternal circulation, but with no difference between preeclampsia patients and controls, and the veno-arterious gradient indicates that the placenta is the source of ADMA. Our study, further, blurs the previously suggested picture of ADMA as an important de- 
terminant of endothelial function in preeclampsia, but does not exclude local or intracellular effects of ADMA and related molecules in the placenta and in the maternal endothelium.

\section{REFERENCES}

1. Sibai B, Dekker G, Kupferminc M 2005 Pre-eclampsia. Lancet 365:785-799

2. Braekke K, Holthe MR, Harsem NK, Fagerhol MK, Staff AC 2005 Calprotectin, a marker of inflammation, is elevated in the maternal but not in the fetal circulation in preeclampsia. Am J Obstet Gynecol 193:227-233

3. Braekke K, Harsem NK, Staff AC 2006 Oxidative stress and antioxidant status in fetal circulation in preeclampsia. Pediatr Res 60:560-564

4. Braekke K, Ueland PM, Harsem NK, Karlsen A, Blomhoff R, Staff AC 2007 Homocysteine, cysteine, and related metabolites in maternal and fetal plasma in preeclampsia. Pediatr Res 62:319-324

5. Irgens HU, Reisaeter L, Irgens LM, Lie RT 2001 Long term mortality of mothers and fathers after pre-eclampsia: population based cohort study. BMJ 323:1213-1217

6. Tenhola S, Rahiala E, Martikainen A, Halonen P, Voutilainen R 2003 Blood pressure, serum lipids, fasting insulin, and adrenal hormones in 12-year-old children born with maternal preeclampsia. J Clin Endocrinol Metab 88:1217-1222

7. Vatten LJ, Romundstad PR, Holmen TL, Hsieh CC, Trichopoulos D, Stuver SO 2003 Intrauterine exposure to preeclampsia and adolescent blood pressure, body size, and age at menarche in female offspring. Obstet Gynecol 101:529-533

8. Nuyt AM 2008 Mechanisms underlying developmental programming of elevated blood pressure and vascular dysfunction: evidence from human studies and experimental animal models. Clin Sci (Lond) 114:1-17

9. Boger RH 2006 Asymmetric dimethylarginine (ADMA): a novel risk marker in cardiovascular medicine and beyond. Ann Med 38:126-136

10. Bode-Boger SM, Boger RH, Kienke S, Junker W, Frolich JC 1996 Elevated $\mathrm{L}$-arginine/dimethylarginine ratio contributes to enhanced systemic NO production by dietary L-arginine in hypercholesterolemic rabbits. Biochem Biophys Res Commun 219:598-603

11. Kielstein JT, Zoccali C 2005 Asymmetric dimethylarginine: a cardiovascular risk factor and a uremic toxin coming of age? Am J Kidney Dis 46:186-202

12. Zsuga J, Torok J, Magyar MT, Valikovics A, Gesztelyi R, Lenkei A, Csiba L, Keki S, Zsuga M, Bereczki D 2007 Dimethylarginines at the crossroad of insulin resistance and atherosclerosis. Metabolism 56:394-399

13. Stuhlinger MC, Stanger O 2005 Asymmetric dimethyl-L-arginine (ADMA): a possible link between homocyst(e)ine and endothelial dysfunction. Curr Drug Metab 6:3-14

14. Holden DP, Fickling SA, Whitley GS, Nussey SS 1998 Plasma concentrations of asymmetric dimethylarginine, a natural inhibitor of nitric oxide synthase, in normal pregnancy and preeclampsia. Am J Obstet Gynecol 178:551-556

15. Pettersson A, Hedner T, Milsom I 1998 Increased circulating concentrations of asymmetric dimethyl arginine (ADMA), an endogenous inhibitor of nitric oxide synthesis, in preeclampsia. Acta Obstet Gynecol Scand 77:808-813

16. Kim YJ, Park HS, Lee HY, Ha EH, Suh SH, Oh SK, Yoo HS 2006 Reduced $\mathrm{L}$-arginine level and decreased placental eNOS activity in preeclampsia. Placenta 27:438-444

17. Maas R, Boger RH, Schwedhelm E, Casas JP, Lopez-Jaramillo P, Serrano N, Diaz LA 2004 Plasma concentrations of asymmetric dimethylarginine (ADMA) in Colombian women with pre-eclampsia. JAMA 291:823-824

18. Savvidou MD, Hingorani AD, Tsikas D, Frolich JC, Vallance P, Nicolaides KH 2003 Endothelial dysfunction and raised plasma concentrations of asymmetric dimethylarginine in pregnant women who subsequently develop pre-eclampsia. Lancet 361:1511-1517
19. Speer PD, Powers RW, Frank MP, Harger G, Markovic N, Roberts JM 2008 Elevated asymmetric dimethylarginine concentrations precede clinical preeclampsia, but not pregnancies with small-for-gestational-age infants. Am J Obstet Gynecol 198:112-117

20. Tsukahara H, Ohta N, Tokuriki S, Nishijima K, Kotsuji F, Kawakami H, Ohta N, Sekine K, Nagasaka H, Mayumi M 2008 Determination of asymmetric dimethylarginine, an endogenous nitric oxide synthase inhibitor, in umbilical blood. Metabolism 57:215-220

21. ACOG Committee on Obstetric Practice 2002 ACOG practice bulletin. Diagnosis and management of preeclampsia and eclampsia. Number 33, January 2002. American College of Obstetricians and Gynecologists. Int J Gynaecol Obstet 77:67-75

22. Johnsen SL, Rasmussen S, Wilsgaard T, Sollien R, Kiserud T 2006 Longitudinal reference ranges for estimated fetal weight. Acta Obstet Gynecol Scand 85:286-297

23. Ueland PM, Midttun O, Windelberg A, Svardal A, Skalevik R, Hustad S 2007 Quantitative profiling of folate and one-carbon metabolism in large-scale epidemiological studies by mass spectrometry. Clin Chem Lab Med 45:1737-1745

24. Bode-Boger SM, Scalera F, Kielstein JT, Martens-Lobenhoffer J, Breithardt G Fobker M, Reinecke H 2006 Symmetrical dimethylarginine: a new combined parameter for renal function and extent of coronary artery disease. J Am Soc Nephrol $17: 1128-1134$

25. Siroen MP, Teerlink T, Nijveldt RJ, Prins HA, Richir MC, van Leeuwen PA 2006 The clinical significance of asymmetric dimethylarginine. Annu Rev Nutr 26:203228

26. Stillman IE, Karumanchi SA 2007 The glomerular injury of preeclampsia. J Am Soc Nephrol 18:2281-2284

27. Ellis J, Wennerholm UB, Bengtsson A, Lilja H, Pettersson A, Sultan B, Wennergren M, Hagberg H 2001 Levels of dimethylarginines and cytokines in mild and severe preeclampsia. Acta Obstet Gynecol Scand 80:602-608

28. Evans RW, Powers RW, Ness RB, Cropcho LJ, Daftary AR, Harger GF, Vergona R, Finegold DN 2003 Maternal and fetal amino acid concentrations and fetal outcomes during pre-eclampsia. Reproduction 125:785-790

29. Herrmann W, Isber S, Obeid R, Herrmann M, Jouma M 2005 Concentrations of homocysteine, related metabolites and asymmetric dimethylarginine in preeclamptic women with poor nutritional status. Clin Chem Lab Med 43:1139-1146

30. Maeda T, Yoshimura T, Okamura H 2003 Asymmetric dimethylarginine, an endogenous inhibitor of nitric oxide synthase, in maternal and fetal circulation. J Soc Gynecol Investig 10:2-4

31. Saarelainen H, Valtonen P, Punnonen K, Laitinen T, Raitakari OT, Juonala M, Heiskanen N, Lyyra-Laitinen T, Viikari JS, Vanninen E, Heinonen S 2008 Subtle changes in ADMA and L-arginine concentrations in normal pregnancies are unlikely to account for pregnancy-related increased flow-mediated dilatation. Clin Physiol Funct Imaging 28:120-124

32. Staff AC, Berge L, Haugen G, Lorentzen B, Mikkelsen B, Henriksen T 2004 Dietary supplementation with L-arginine or placebo in women with pre-eclampsia. Acta Obstet Gynecol Scand 83:103-107

33. Meher S, Duley L 2007 Nitric oxide for preventing pre-eclampsia and its complications. Cochrane Database Syst Rev CD006490

34. Rytlewski K, Olszanecki R, Korbut R, Zdebski Z 2005 Effects of prolonged oral supplementation with L-arginine on blood pressure and nitric oxide synthesis in preeclampsia. Eur J Clin Invest 35:32-37

35. Rytlewski K, Olszanecki R, Lauterbach R, Grzyb A, Basta A 2006 Effects of oral L-arginine on the foetal condition and neonatal outcome in preeclampsia: a preliminary report. Basic Clin Pharmacol Toxicol 99:146-152

36. Lopez-Jaramillo P, Garcia RG, Lopez M 2005 Preventing pregnancy-induced hypertension: are there regional differences for this global problem? J Hypertens 23:1121-1129

37. Vida G, Sulyok E, Ertl T, Martens-Lobenhoffer J, Bode-Boger SM 2007 Plasma asymmetric dimethylarginine concentration during the perinatal period. Neonatology 92:8-13 